\title{
CHALLENGES AND VARIABLES AFFECTING THE SUCCESS OF SENTINEL LYMPH NODE BIOSPY IN MALIGNANT MELANOMA: A 4-YEAR ANALYSIS OF CLINICAL RESULTS
}

\section{Dobromir D. Dimitrov, Veselin I. Kirov', Borislav A. Ignatov ${ }^{2}$, Boris D. Tsankov², Hyuliya E. Feradova ${ }^{2}$, Vasya Vutova ${ }^{2}$, Silviya P. Hristova ${ }^{2}$, Vasil D. Nanev, Genoveva B. Valcheva, Tashko S. Deliyski}

Department of Surgical Oncology, Medical University - Pleven ${ }^{1}$ Consultative-Diagnostic Oncology Center, Medical University - Pleven ${ }^{2}$ Student Study-Research Group of Surgical Oncology, Medical University - Pleven, Bulgaria

\author{
Corresponding Author: \\ Borislav A. Ignatov \\ Department of Surgical Oncology \\ University Hospital "Dr George Stranski" \\ 8A, George Kotchev' str. \\ Pleven, 5800 \\ Bulgaria \\ e-mail: b_ignatov32@yahoo.com
}

Received: July 01, 2013

Revision received: December 09, 2013

\section{Summary}

In the last 15 years, sentinel lymph node biopsy (SLNB) for patients with malignant melanoma (MM) has been introduced into the clinical practice. Our aim was to make a retrospective analysis of clinical results in order to assess the success ratio of SLNB and the variables affecting it in MM patients, treated in the Oncology Center at the University Hospital "Dr George Stranski" - Pleven, Bulgaria for a 4-year period. A research index card was used to process the oncologic files of 82 out of 102 patients with MM, treated and monitored at the Oncology centre between 01.01.2008 and 31.12.2011. TNM-stages, Breslow and Clark levels, location, characteristics of the skin lesion, diagnostic excision, type of lymph node dissection, number of SLN, localization of SLN, etc. were registered in the index card. SLNB staging was carried out in 28 patients. Sentinel lymph nodes were found in 22 of them. The success ratio of the method was $78.6 \%$. Histologically, metastatic SLN were found in 4 cases. The Patent Blue Dye method was used in 22 of the cases $(78 \%)$. A combined radiocolloid and dye method was used in 5 cases (18\%). A radiocolloid method was used in 1 case $(4 \%)$ only. Our research showed that more therapeutic lymph node dissections were performed in cases of locally advanced MM, which in turn worsened the clinical results. According to our research, the SLNB success ratio depends on the precision of diagnostic excision $(\mathrm{p}=0.019)$, lesion location $(p=0.015)$, Clark level $(p=0.0229)$, mapping method $(\mathrm{p}=0.08)$ and the type of melanoma $(\mathrm{p}=0.088)$.

Key words: malignant melanoma, retrospective analysis, sentinel lymph node biopsy, lymph node dissection

\section{Introduction}

Epidemiologically, there is a significant difference in the incidence of malignant melanoma (MM) around the world. It is 10 times more common in Australia, New Zealand, as compared to Europe [1]. The number of MM cases in Bulgaria has increased three times over the last 30 years $(0.5 / 100.000$ in 1980 to $1.6 / 100.000$ in 2010) but it still remains a rare disease [2]. The global tendency shows a growth in the number of reported cases and reveals the social significance of the disease due to high mortality rates of patients. In the last 20 years, the algorithm for 
diagnosis, staging and treatment of melanoma has undergone significant changes. The introduction of sentinel lymph node biopsy (SLNB) in clinical practice has made it possible to reduce regional lymph node dissections (LND) and subsequent complications and improve staging and defining the prognosis of patients as well as the involvement of adjuvant therapy [3, 4].

The clinical types of MM are as follows: lentigo maligna, lentigo maligna melanoma, superficial spreading melanoma, acral lentiginous melanoma, mucosal melanoma, nodular melanoma, polypoid melanoma, desmoplastic melanoma, amelanotic melanoma, soft-tissue melanoma. [5]

The T category of the TNM staging of MM lesions is defined by Clark level and Breslow thickness. Clark level describes five levels of anatomical invasion of the melanoma in the skin from the epidermis through the papillary and reticular dermis to subcutaneous fat. Breslow thickness, determined directly by ocular micrometer (and measured in SI millimeters) shows the depth of invasion of tumor cells down from the granular layer of the epidermis. Melanomas less than $0.76 \mathrm{~mm}$ are classified as very thin, melanomas from 0.76 to $1.5 \mathrm{~mm}$ are thin, melanomas $1.5-3.0 \mathrm{~mm}$ are intermediate, and thick melanomas are over $3.0 \mathrm{~mm} \mathrm{[6].} \mathrm{The}$ most important prognostic factor in $\mathrm{MM}$ is Breslow thickness and ulceration status. The measurement of the Breslow thickness is most accurately done by diagnostic excision [7].

Today, an interdisciplinary approach to MM diagnosis and treatment is applied. For almost 20 years, patients with intermediate or thick primary melanomas (Breslow's depth over $1.5 \mathrm{~mm}$ ) have been advised to undergo sentinel lymph node biopsy (SLNB) and this procedure has become a standard approach $[8,9]$. Our aim was to make a retrospective analysis of clinical results in order to assess the success ratio of SLNB and the variables affecting it in MM patients, treated in the Oncology Center at the University Hospital "Dr George Stranski" - Pleven, Bulgaria in a 4year period.

\section{Materials and Methods}

A research index card was used to process the oncologic files of 82 out of 102 patients with MM, treated and monitored at the Oncology Center at the University Hospital "Dr George Stranski" Pleven, Bulgaria between 01.01.2008 and 31.12.2011. Medical documentation was missing from the files of 20 patients. The data was processed and analysed by the student studyresearch group of Surgical Oncology at the Medical University-Pleven for 2 months. Age, sex, TNM-stages, Breslow and Clark levels, location, characteristics of the skin lesion, diagnostic excision, lymph node dissection type, number of SLN, localization of SLN, postoperative chemotherapy, imaging data etc. were registered in the index card.

It was found that the most common location of the skin lesion was on the back - 23 cases (28\%). The prevalent type of lesion was the nodular form - 28 cases $(28.05 \%)$. Distribution according to the location of the lesion in our research is demonstrated in Figure 1. The predominant site was the back, followed by the upper and lower limbs. The distribution of the melanomas according to their type is shown in Figure 2. Data was collected about the size of the malignant lesions (Figure 3) which showed that most of them were less that $3 \mathrm{~cm}$. All the cases were histologically proven. Breslow and Clark level were measured by pathologists.

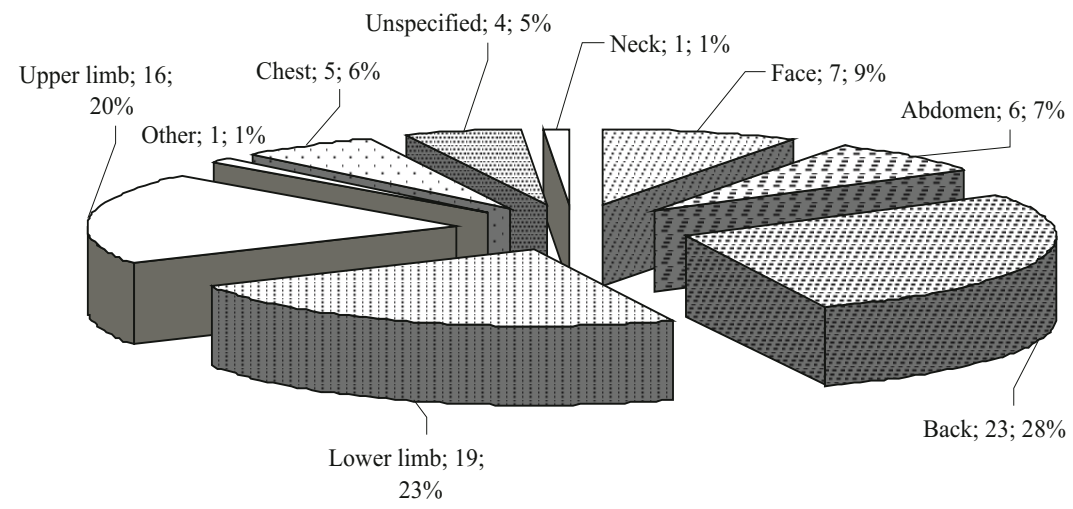

Figure 1. Distribution according to lesion location 

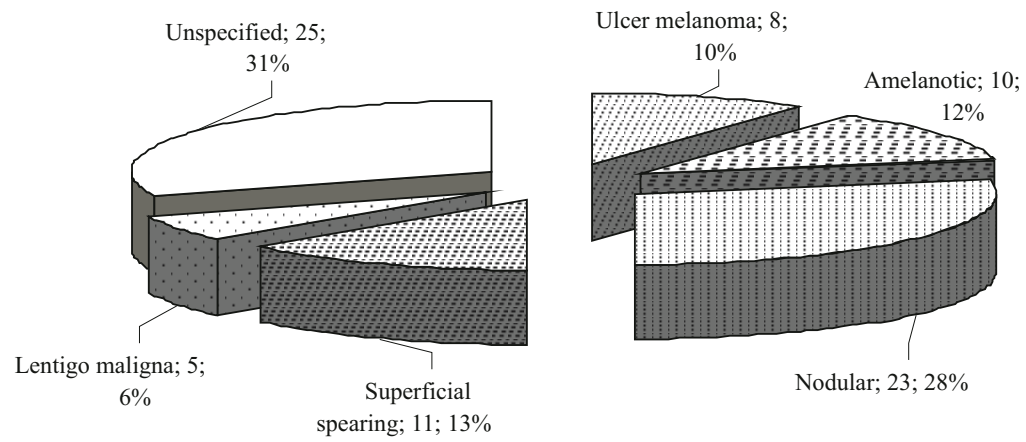

Figure 2. Distribution according to the type of melanoma
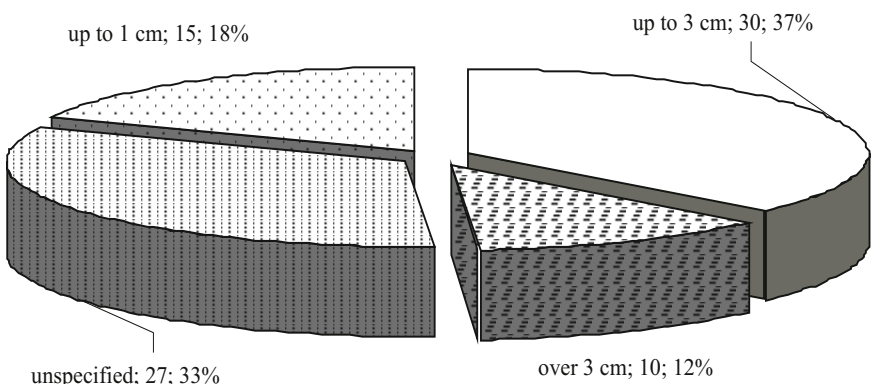

Figure 3. Distribution according to lesion size

The algorithm of diagnosis and treatment (Figure 4) in the Department of Surgical Oncology was based on the interdisciplinary approach to melanoma. Dermatologists, surgical oncologists, medical oncologists and nuclear medicine physicians took part in the process of diagnosing and treatment. SLNB was carried out using the methods of lymphoscintigraphy, dyeing with Patent Blue V, or a combined method. Seventeen to eighteen hours prior to SLNB, a radiocolloid was injected intradermally around the diagnostic excision scar by a nuclear medicine specialist. During the surgical operation, $2 \mathrm{ml}$ of Patent Blue V was injected intradermally in the same area around the scar. A skin incision above the regional lymph node basin was made 10-15 minutes after the blue dye injection. All blue-dyed or radioactive lymph nodes were harvested for histologic examination.

The success ratio of the SLNB was calculated with a standard formula, and data was analysed with the Statgraphics plus program. The statistical methods used were alternative analysis, analysis of variance, graphical analysis and correlation analysis.

\section{Results}

In 37 of the cases (45.1\%), Breslow thickness was 1 to $3 \mathrm{~mm}$. In the same patients Clark levels were up to 3 to 4 . Out of all the patients, 43 (52.4\%) were with locally advanced T3 and T4 stage cases. The distribution of patients according to Breslow thickness and Clark level is shown on Figure 5 and Figure 6.

The diagnostic excision was carried out in the Oncology Centre in 46 cases $(56 \%)$, in other clinics - in 33 cases $(40 \%)$ and 3 patients $(4 \%)$ had not undergone diagnostic excision. SLNB staging was performed in 28 cases. Sentinel lymph nodes were found in 22 of the patients. Respectively, the success ratio of the method was $78.6 \%$. The mapping methods used were: Patent blue $\mathrm{V}$ alone -22 patients (78\%), radiocolloid and Patent Blue V - 5 (18\%), and radiocolloid alone in one patient (4\%). Histologically, metastatic SLN were found in 4 cases (Table 1). These patients underwent therapeutic lymph node dissection (TLD), and TLD as a procedure of first choice was performed in 9 other cases, i.e. the TLDs were 13 altogether.

In our research, the cases with lesions on the upper limb, lower limb and abdomen had a 100\% successful SLNB - SLN were found in all 11 


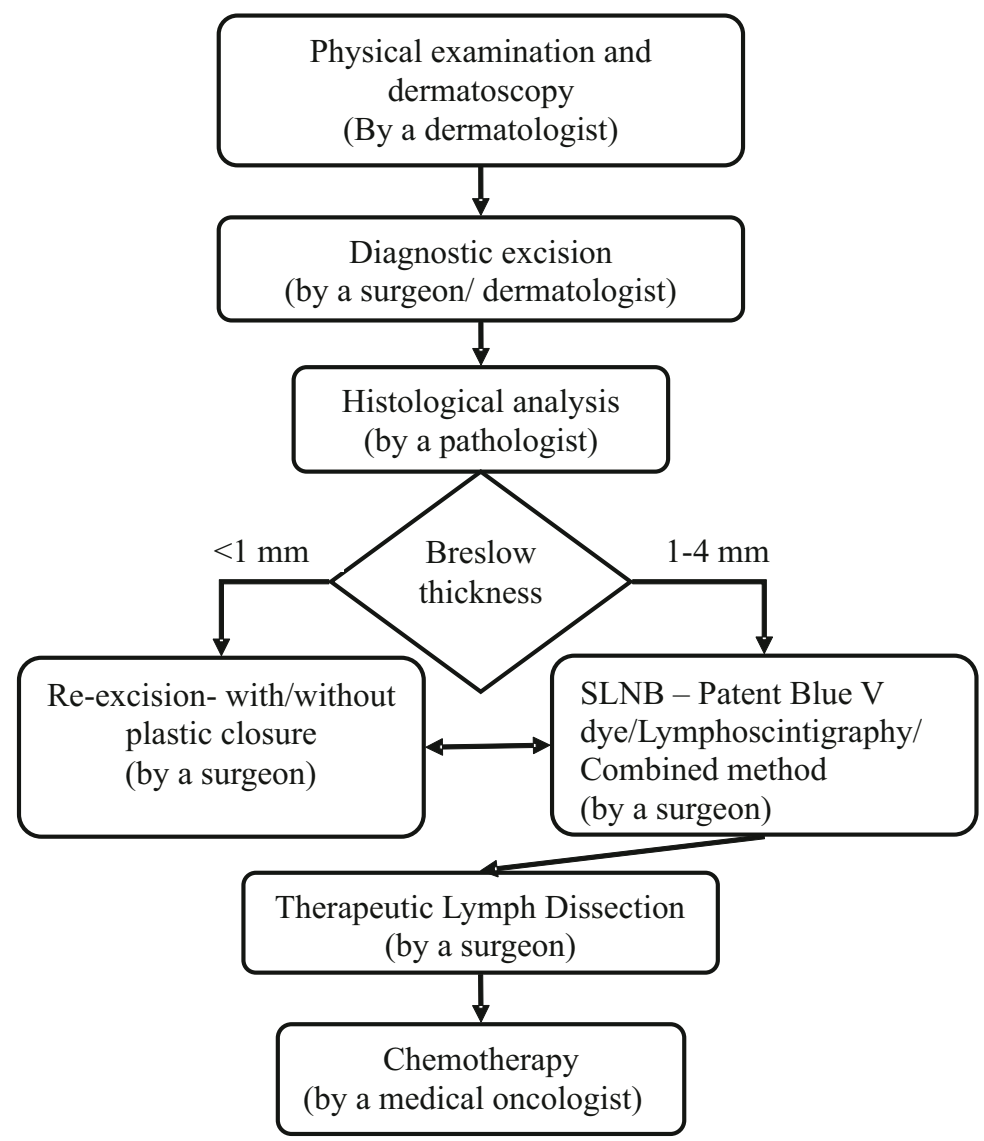

Figure 4. Interdisciplinary approach to malignant melanoma

cases. On the other hand, cases with face, neck and back lesions had SLNB with a lower success ratio. SLNs were found in $72 \%$ of the patients with back lesions ( 10 out of 14 ), in $50 \%$ of those with face lesions ( 1 out of 2 cases) and there were no SLNs found in the case with a neck lesion.

Histology of the SLNs showed a close association with the lesion size $(\mathrm{p}=0.07)$.

Our research showed that the success ratio of
SLNB significantly correlated with the lesion location $(p=0.015)$, Clark level $(p=0.0229)$, the type of melanoma $(\mathrm{p}=0.088)$ and as well as with the mapping (radiocolloid or Patent Blue V) method $(p=0.08)$. The mapping method was determined by the location of the lesion $(p=0.0008)$. The lower success ratio of SLNB in our study $(78.6 \%)$ closely correlated with the precision of the diagnostic excision $(p=0.019)$.

Table 1. Results of sentinel lymph node biopsy SLNB

\begin{tabular}{llll}
\hline SLN found & & Histology & \\
\hline Yes & No & Metastatic & Non - metastatic \\
\hline 22 cases & 6 cases & 4 cases & 18 cases \\
\hline $78.6 \%$ & $21.4 \%$ & $18.2 \%$ & $81.8 \%$ \\
\hline
\end{tabular}




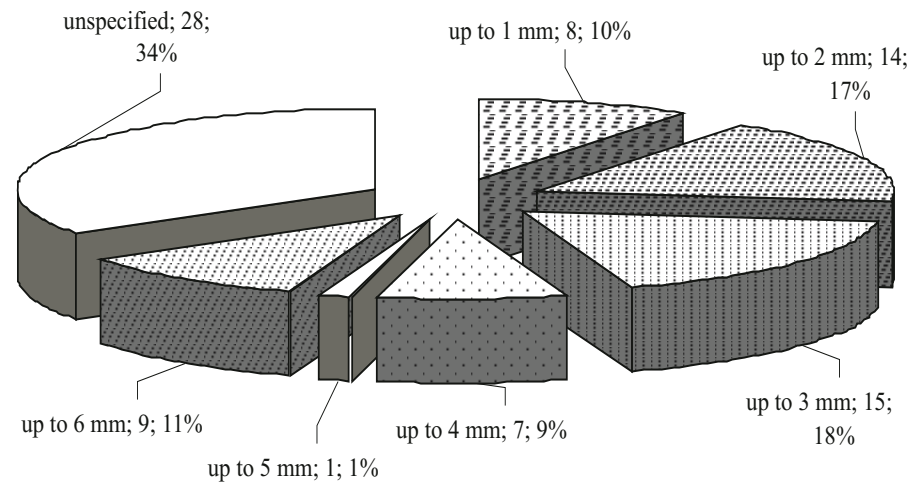

Figure 5. Distribution according to Breslow thickness

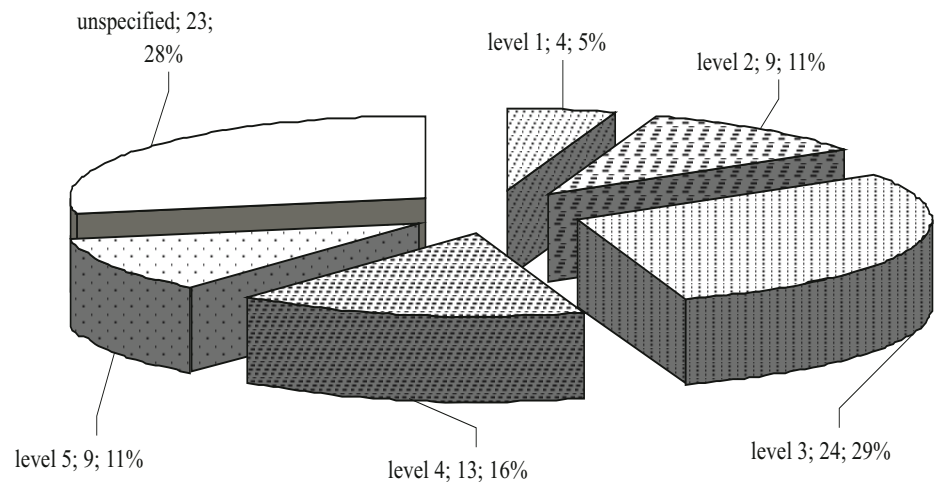

Figure 6. Distribution according to Clark level

\section{Discussion}

In our research, the number of patients with locally advanced MM was higher than the one reported in the literature. This suggests that it is necessary to perform more lymph node dissections during the staging and treatment of patients with MM, which in turn worsens the clinical results. The large number of unspecified lesions is due to patients referred to our clinic from other hospitals where such an assessment had not been performed.

The success ratio reported in the literature is about $92 \%$ [10-12]. In our research, the success ratio of the method was $78.6 \%$. Excisions performed at non-specialized clinics and medical doctors lead to a lower success ratio of SLNB in our study $(\mathrm{p}=0.019)$. A possible reason for this result is that the old guidelines requiring a wide diagnostic excision and simultaneous large lymph node dissection at the region are still followed by most surgeons. In a study with 1563 patients, McKenna at al. compared surgical management of cutaneous melanoma carried out by dermatologists, surgeons and general practitioners. These authors found that general surgeons used wider excision margins as compared with the other specialists, while the dermatology group achieved better results regarding survival rate and recurrence-free interval, as compared to the general and plastic surgery groups [10]. Our research showed that the success of SLNB had a significant correlation with the lesion location $(p=0.015)$, Clark level $(p=0.0229)$, the type of melanoma $(p=0.088)$ and the mapping (radiocolloid or Patent Blue V) method $(p=0.08)$. The latter two correlations have a p-value less than 0.1 , so we cannot accept the hypothesis according to which the criteria are independent at a $90 \%$ confidence level. Morton at al. and Bostik et al. have come to a similar conclusion, stating that lymphatic mapping with blue dye plus radiocolloid is more successful than using blue dye alone [11, 12] . 
According to Morton at al., successful SLN identification is possible using a standardized method and a multidisciplinary approach (surgery, nuclear medicine, and pathology) by centers worldwide [11].

According to our study the mapping method was dependent on the location of the lesion $(p=0.0008)$. Patent blue $\mathrm{V}$ was enough to easily map lymphatic paths of the limbs and the abdomen, while Tc Nanocoll 99m was needed in cases of face, neck and back lesions. This was relevant to the findings of different authors who worked on potential metastatic sites in cases of cutaneous head and neck melanoma, using lymphoscintigraphy $[13,14]$.

\section{References}

1. Wikipedia, the Free Encyclopedia [Internet]. Melanoma. [cited 2013Jan 18]. Available from: http://en.wikipedia.org/wiki/Melanoma

2. Dimitrova N, Vukov M, Valerianova Z, editors. Cancer incidence in Bulgaria 2009. Vol XX. Bulgarian National Cancer Registry: Publisher “AVIS-24” Ltd.; 2011.

3. North JH Jr, Spellman JE. Role of sentinel node biopsy in the management of malignant melanoma. Oncology (Huntington). 1996;10(8): 1237-42.

4. Lingam MK, Mackie RM, McKay AJ. Intraoperative identification of sentinel node in patients with malignant melanoma. Br J Cancer. 1997;75(10):1505-8.

5. James WD, Berger TG, Elston DM. Melanocytic nevi and neoplasms. In: Andrews' Diseases of the Skin: Clinical Dermatology. 11th ed. Saunders Elsevier; 2011.p. 675-93.

6. Sanchez RL, Raimer S. Pigmentary disturbances and melanocytic tumors. In: Sanchez RL, Raimer $\mathrm{S}$, editors. Vademecum Dermatopathology. Georgetown, Texas: Landes Bioscience: 2001. p. 270-85.

7. Marghoob AA, Koenig K, Bittencourt FV, Kopf AW, Bart RS. Breslow thickness and Clark level in melanoma: support for including level in pathology reports and American Joint Committee on Cancer Staging. Cancer. 2000;88(3):589-95.

8. Testoril A, Rutkowski P, Marsden J, Bastholt L, Chiarion-Sileni V, Hauschild A, et al. Surgery and radiotherapy in the treatment of cutaneous melanoma. Ann Oncol. 2009;20(Suppl 6):vi22-vi29.

\section{Conclusion}

The analysis covered the main criteria in the diagnosis and treatment of malignant melanoma. It showed that the diagnostic excision must be restricted and only performed by specialists in surgical oncology or oncodermatology. According to our research, the SLNB success ratio significantly correlates with the diagnostic excision preciseness $(\mathrm{p}=0.019)$, lesion location $(\mathrm{p}=0.015)$, Clark level $(\mathrm{p}=0.0229)$, mapping method $(\mathrm{p}=0.08)$ and type of melanoma $(p=0.088)$. Cases with face, neck and back lesions had SLNB with lower success ratio. We highly recommend the use of radionuclide method for these low success areas, especially by teams with little experience.

9. Jakub JW, Pendas S, Reintgen DS. Current Status of Sentinel Lymph Node Mapping and Biopsy: Facts and Controversies. Oncologist. 2003;8(1): 59-68.

10. McKenna DB, Marioni JC, Lee RJ, Prescott RJ, Doherty VR. A comparison of dermatologists', surgeons' and general practitioners' surgical management of cutaneous melanoma. $\mathrm{Br} \mathrm{J}$ Dermatol. 2004;151(3):636-44.

11. Morton DL, Thompson JF, Essner R, Elashoff R, Stern S, Nieweg O, et al. Validation of the accuracy of intraoperative lymphatic mapping and sentinel lymphadenectomy for early-stage melanoma. Ann Surg. 1999;230(4):453-63.

12. Bostick P, Esner R, Glass E. Comparison of Blue Dye and Probe-Assisted Intraoperative Lymphatic Mapping in Melanoma to Identify Sentinel Nodes in 100 Lymphatic Basins. Arch Surg. 1999; 134(1):43-9.

13. O'Brien CJ, Uren RF, Thompson JF, HowmanGiles RB, Petersen-Schaefer K, Shaw HM, et al. Prediction of potential metastatic sites in cutaneous head and neck melanoma using lymphoscintigraphy. Am J Surg. 1995;170(5):461-6.

14. Uren RF, Howman-Giles R, Thompson JF. Patterns of Lymphatic Drainage from the Skin in Patients with Melanoma. J Nucl Med. 2003;44(4):570-82. 